\title{
Kernos
}

Revue internationale et pluridisciplinaire de religion grecque antique

$20 \mid 2007$

Varia

\section{PARKER Robert, Polytheism and Society at Athens}

\section{Vinciane Pirenne-Delforge}

\section{(2) OpenEdition}

\section{Journals}

Édition électronique

URL : https://journals.openedition.org/kernos/359

DOI : $10.4000 /$ kernos.359

ISSN : 2034-7871

Éditeur

Centre international d'étude de la religion grecque antique

Édition imprimée

Date de publication : 1 janvier 2007

Pagination : 425-428

ISSN : 0776-3824

Référence électronique

Vinciane Pirenne-Delforge, « PARKER Robert, Polytheism and Society at Athens », Kernos [En ligne], 20 |

2007, mis en ligne le 17 mars 2011, consulté le 08 septembre 2022. URL : http://

journals.openedition.org/kernos/359; DOI : https://doi.org/10.4000/kernos.359 
somewhat strained when trying to match the two categories. The evidence used to elucidate Pindar and the persons whom the odes honour is drawn from a wide variety of sources as to date and character. In view of some of these authors being substantially later than Pindar, a consideration of the methodological implications of such a wide use would have been appropriate. For example, the conclusion that Neoptolemos had hero-cult in Delphi already in the Classical period rests on the dismissal of Pausanias' statement of a 3rd-century introduction of the cult as a misunderstanding. If Pausanias got it wrong here, can we really trust all his other information without discussing it? The same goes for sources like Lucian or Johannes Lydos, not to mention the scholia, which figure prominently. One concept frequently referred to is the notion of 'popular belief, which is used to explain what kind of religious sentiment we may encounter in the sources. It would have been interesting to have this notion defined, considering the often selective and elitist nature of many ancient authors.

Finally, if we are to assume that heroization of ordinary persons, after death and sometimes even in lifetime, was a fairly common event in Classical Greek society, we have to consider the contents of this cult. What kind of ritual was performed for these heroes, the same kind as for the gods and mythic or epic heroes, that is thysiai with animal sacrifice, or was the cult more like the cult of the dead? If the religious attention paid to them did not contain animal sacrifice, can we label it a 'hero-cult' or 'heroic honours'? These are highly complex issues and C. has made it easy for himself by avoiding them by simply speaking of 'hero-cult', 'cult' or 'heroization' without really addressing the topic of the ritual content or the religious consequences of his extension of the practice of heroization in the Classical period.

To conclude, any study, which questions traditional scholarly notions of what went on in Greek religion, is refreshing. Hero-cults, and its different facets, have always been a difficult item to digest and understand for modern scholars, partly since there is no good equivalent for comparison within our Judeo-Christian culture (the saints being fundamentally different from the Greek heroes). C.'s study has certainly opened up new ways of thinking about both the purpose and the institution of hero-cults, which will hopefully stimulate yet further work on Greek heroes.

Gunnel Ekroth

(University of Stockholm)

PARKER Robert, Polytheism and Society at Athens, Oxford, Oxford University Press, 2005. 1 vol. $14 \times 22 \mathrm{~cm}$, XxxII+544 p. (Oxford Classical Monographs). ISBN : 0-19-927483-5.

En 1996, R. Parker (R.P.) publiait Athenian Religion. A History, un ouvrage qui tentait le pari difficile d'inscrire les manifestations de la vie religieuse athénienne dans une perspective chronologique qui en identifie les évolutions et les changements. Le pari était réussi, mais il avait un prix : la mise à l'écart d'un traitement circonstancié d'éléments essentiels de cette vie religieuse dont la dimension historique, au sens chronologique du terme, peinait à rendre compte. On attendait donc la suite... Elle est arrivée sous la forme d'un fort ouvrage, absolument remarquable: la connaissance approfondie de la documentation, qu'elle soit littéraire, épigraphique, archéologique ou iconographique, et le traitement adéquatement critique d'une bibliographie vraiment internationale s'y soutiennent et interagissent pour fournir une synthèse qui fera date, tant pour les dossiers qui s'y déploient que pour les questionnements précis et la méthode pragmatique ${ }^{1}$ qui en structurent l'analyse.

${ }^{1}$ Je transpose ainsi ce que R.P. appelle, à la page 222, « a measure of everyday canniness and caution »... 
Une première partie aborde en sept chapitres ce que j'appellerai «l'armature» du système, à défaut de disposer d'un titre choisi par l'A. lui-même. Il s'agit d'identifier les cadres de la vie religieuse et ses acteurs. Ces cadres sont l'oikos (chap. 1), les différents cercles de sociabilité (chap. 2), le dème et la cité elle-même (chap. 3, qui pose la question fondamentale du «où » les dieux sont placés), avec, notamment, la scène du théâtre de Dionysos (chap. 7, où la lucidité flirte avec l'humour!), sans oublier le contexte «international », qui surgit dès que l'on dépasse les frontières de l'Attique (chap. 4). Les acteurs sont «ceux qui prient pour Athènes" (chap. 5), mais aussi «ceux qui font des rites un métier» (chap. 6) et emmènent le propos du côté des limites où s'entrecroisent les notions de magie et de religion. Chaque dossier est traité avec une grande lucidité et les questions posées secouent bon nombre d'idées reçues. Je prendrai deux exemples tirés du 1er chapitre, mais il pourrait s'en ajouter bien d'autres auxquels je ne renonce qu'à regret! Le premier touche à l'bestia domestique et à la déesse qui se profile derrière son nom. Dans notre vision de la maisonnée grecque, ce foyer est le cœur intangible et le signe religieux de la continuité familiale. Or les fouilles peinent à identifier une structure permanente de ce type dans les maisons et une conclusion plausible serait de faire de ce symbole de la permanence une structure transportable et mobile! Le symbole ne s'en trouve pas nécessairement invalidé, mais nous devons assurément en nuancer la littéralité (p. 9-15). Deuxième exemple : les prétendus « household gods ». Aucun texte classique ne parle ainsi des dieux honorés dans la maisonnée. En revanche, les $\pi \alpha \tau \varrho \tilde{\omega} O \iota$ $\theta \varepsilon o i ́$ sont omniprésents. Ils intègrent les dieux de l'oikos au cercle des autres divinités «patrimoniales» des différents groupes de sociabilité auxquels chaque Athénien appartient. C'est autour de leurs autels que s'opère la « socialization into the religion of the city » (p. 23). Remarquons aussi à quel point les dieux honorés dans l'oikos sont peu anthropomorphes : "Hestia is the hearth, Zeus Ktesios a pot, Apollo Aguieus a pillar, Hermes a block with head and phallus; Zeus Herkeios had an altar, but was perhaps not further represented. A strange collection they would make, lined up in a row! » (p. 19). Serait-ce, comme l'affirme Fr. Frontisi, parce qu'ils vivent précisément près des hommes que leur altérité se doit d'être soulignée ? R.P. qualifie le propos d'« elegant explanation ", mais l'adopte-t-il pour autant? Dans le monde sublunaire qu'il assume (p. 5) l'expression fait évidemment penser à Paul Veyne - on sent bien qu'une certaine "élégance » à la française est perçue dans toutes ses implications, avec sympathie, mais qu'elle est aussi mise à distance. Je reviendrai sur ce point.

La deuxième partie couvre un peu moins de la moitié de l'ouvrage et prend le relais des Attische Feste de L. Deubner (1932) que n'avaient remplacé ni les Festivals of the Athenians de H.W. Parke (1977) ni les Festivals of Attica de E. Simon (1983). Il ne s'agit pas pour autant de livrer un parcours analytique de l'année liturgique athénienne qui rassemblerait la documentation disponible en tentant de dessiner le profil de chaque fête (une « check list » est donnée en annexe). Ce genre d'exercice est précisément celui auquel se sont attelées les études antérieures. L'approche est ici plus englobante, surtout dans les quatre premiers chapitres. Ils abordent respectivement la question des célébrants (chap. 8) - avec la question frustrante du degré de participation des femmes aux différentes fêtes qui ne leur sont pas directement destinées -, la détermination des types d'activités accomplies lors des fêtes (chap. 9), l'épaisseur qualitative de l'année liturgique dans ses différents rythmes et cycles (chap. 10) et la place des parthenoi au sein des activités rituelles (chap. 11). Les garçons ont fait une apparition ici et là au fil des chapitres précédents et ils reçoivent aussi une annexe sur le gymnase à la fin de ce chapitre sur les filles. La justification de ce chapitre sur les parthenoi tient à l'abondance de la documentation qui voit les filles mises en scène par la cité (cf. déjà La fille d'Athènes de Pierre Brulé, un livre cité dès la première note de ce chapitre), mais aussi au fait que, dans leur cas, on perçoit une forme d'initiation, dont il convient d'élucider la 
portée. Comme on pouvait s'y attendre, seule l'arkteia de Brauron entre dans cette catégorie problématique, mais avec toute la prudence et la nuance dont R.P. est capable.

Les quatre chapitres suivants de cette partie abordent des fêtes singulières et des ensembles cohérents : les Panathénées (chap. 12), les « fêtes de femmes » que sont les Thesmophories et les Adonies (chap. 13), les Anthestéries et d'autres rituels dionysiaques (chap. 14), les fêtes éleusiniennes (chap. 15) - avec une belle analyse des Mystères ( $\ldots$... the all-welcoming values of Eleusinian Deo hosted the most open of all Greek cults, one which illustrates one of the senses in which there was such a thing as 'Greek religion'», p. 342) et de la vision de l'au-delà qu'ils impliquent («We see here very clearly the difference which divides a religion that offers hopes from one which imposes firm beliefs », p. 368). Le dernier chapitre de cette $2^{\mathrm{e}}$ partie sert de conclusion à l'ensemble, mais ouvre surtout la discussion à la notion de « rituel » qui est finalement plus centrale que celle de fête dans ces différents chapitres. La discussion tourne le dos à toute théorie unifiante pour privilégier la nuance qui fait de chaque fête un ensemble particulier dont la portée est collective et qui prescrit les rôles sociaux de ses participants. C'est également ici que se pose la question des cultes électifs, dont un bon nombre nous échappe, et celle de la relation entre les mythes et les fêtes (une articulation dont un tableau récapitulatif est donné à la fin du chapitre). À la première, R.P. répond que les expériences vécues dans les cultes choisis devaient être familières : différence de degré et non de nature entre cultes publics et associations privées. À la deuxième, il oppose la grande variété des situations, pour se pencher davantage sur le temps des fêtes luimême, qui est moins le temps primordial rêvé par Eliade que celui des émotions partagées et d'une expérience vécue où le lien entre passé et présent est plus ou moins étroit selon les cas.

La $3^{\mathrm{e}}$ et dernière partie compte deux chapitres qui portent le même titre : Gods at Work I et II. Et voici qu'arrive le polytheism du titre de l'ouvrage, en un constat liminaire peu engageant : «Greek polytheism is indescribable » (p. 387). Toutefois, le structuralisme appliqué au monde des dieux grecs fut une manière de « décrire l'indescriptible ». R.P. affronte donc les acquis et les limites de ces interprétations, tout imprégnées de cette "élégance » à la française que j'évoquais plus haut. L'analyse est pertinente et bien informée des infléchissements apportés par M. Detienne à l'approche dumézilienne des polythéismes². Ainsi, il souligne, après Detienne, les risques de figer le profil d'un dieu grec en appliquant mécaniquement les principes du mode d'action et d'intervention spécifique dans différents champs d'action, alors qu'il s'agissait de sortir de la logique du portrait canonique des encyclopédies. Quelques dieux sont appelés à la barre pour illustrer la critique et on sent bien que la recherche d'une «logique du système» est considérée avec un certain scepticisme. Certes, R.P. a tout à fait raison d'affirmer qu'un panthéon local n'est pas le produit d'une construction logique comme l'est la Théogonie d'Hésiode et que la distribution des compétences en matière de protection divine peut parfois sembler inconsistante (p. 394). Mais quelle était la marge de manœuvre des fidèles dans les choix qu'ils opéraient? La réponse à cette question est un idéal sans doute globalement inaccessible, mais l'apport de chaque historien de la religion grecque réside dans la manière de la poser. L'étude d'un panthéon local déterminé est assurément une des voies d'accès les plus rigoureuses à la compréhension du système. Elle permet de contourner le caractère irréalisable de cet idéal qui viserait à comprendre toutes les interactions entre tous les dieux grecs, sans parler des héros... Au sein de cette approche locale - puisqu'ici c'est bien d'Athènes qu'il s'agit - R.P. a choisi la voie fonctionnelle. Le premier des deux chapitres finaux (chap. 17) regroupe les dieux protecteurs - au sens le plus large du terme - de la cité et de ses habitants, le second

\footnotetext{
${ }^{2}$ Entre autres dans « Expérimenter dans le champ des polythéismes », Kernos 10 (1997), p. 57-72.
} 
(chap. 18) rassemble les dieux qui veillent à la croissance des plantes et des humains - avec une annexe sur les héros et héroïnes. Ces groupements permettent de voir différents dieux en action dans des champs d'action semblables et illustrent bien la difficulté de déterminer des spécificités. Le cas des diverses figures de Zeus comme "pourvoyeur de prospérité » est exemplaire (p. 421-425). Comment ordonner Zeus Herkeios, Ktesios, Meilichios dans un schéma fonctionnel différentiel ? À cette occasion, la notion de «chthonien» est battue en brèche dans une critique pertinente, et la conclusion, prudente, est faite de "peut-être » : «If Zeus Ktesios was concerned both with safe husbanding of the household's property and with extending it, one may wonder what more there was for Zeus Meilichios to contribute. Perhaps Zeus Ktesios was an emblem of prudent management, while Zeus Meilichios controlled more incalculable factors. Perhaps it was a matter of the different groups within which the two gods were honoured. And perhaps the difference was no more clear to Athenians than to us » (p. 425). Dans cet ensemble complexe et fluide qu'est le polythéisme, R.P. reconnait que « the key structuralist postulate that, in such cases, each god brings to the same problem a different expertise is often convincing, and always the best working hypothesis » (p. 445), mais il est des noyaux durs (" hard cases») et des questions insolubles sur la réalité du consensus auquel le savoir partagé par les Grecs pouvait conduire différents individus dans une quête de protection similaire. Il n'est jamais de réponse unique à une aspiration et, dans ce sens-là, le système n'est pas « logique ».

Je concluerai en recommandant chaleureusement la lecture de cet ouvrage à quiconque s'intéresse à la religion grecque. Car, si, comme R.P. l'écrit, « it is difficult to read a sacrificial calendar or a few pages of Pausanias without bewilderment» (p. 445), sa synthèse monumentale ouvre quantité de pistes pour atténuer ce «trouble». Quand une grande rigueur, doublée d'une parfaite connaissance des données anciennes et modernes, se met au service d'une grande probité intellectuelle, cela donne un grand livre.

Vinciane Pirenne-Delforge (FNRS - Université de Liège)

DestréE Pierre, Smith Nicolas D. (éds), Socrates' Divine Sign: Religion, Practice, and V alue in Socratic Philosophy, in Apeiron 38 (2005). ISSN : 0003-6390.

Chacun des dix chapitres de cet ouvrage collectif correspond à une communication du colloque international sur le daimonion socratique du Centre d'Études des Religions et de la Laïcité de l'Université libre de Bruxelles (décembre 2003). Le daimonion de Socrate est évidemment toujours discutable et discuté, mais rares sont les études qui l'ont placé au centre de leur intérêt. Cet ouvrage présente un recueil précieux d'analyses et de réflexions pour ceux qui désirent avoir une image globale des divers avis et des débats les plus importants autour de ce sujet. Les auteurs des essais défendent des points de vue diversifiés, et nous savons gré aux éditeurs d'avoir respecté cette pluralité d'opinions, parfaitement représentative de la complexité du dialogue sur la compréhension du daimonion, un débat commencé déjà parmi les disciples de Socrate. Nous présenterons ici un bref aperçu critique de chacun des chapitres, en insistant sur l'une ou l'autre de leurs analyses, riches et détaillées dans leur totalité, de manière forcément sélective.

Luc Brisson, «Socrates and the Divine Signal According to Plato's Testimony: Philosophical Practice as Rooted in Religious Tradition» (p. 1-12). L'A. explore les textes platoniciens considérés comme authentiques où se présente le "signe divin » (to daimonion sêmeion) de Socrate, afin de décrire ce phénomène et de discuter sa compatibilité avec l'«autonomie morale ", entendue dans le sens kantien de la détermination des actes de l'individu uniquement par son propre raisonnement. D'après L. Brisson, le daimonion sêmeion (le terme to daimonion étant accepté seulement comme un adjectif, synonyme du to theion ou de l'expres- 